\title{
PROPORTION AND CHARACTERISTICS OF NEW PATIENTS WITH CENTRAL SENSITIZATION PRESENTING TO A CHIROPRACTIC PRACTICE - A CROSS-SECTIONAL OBSERVATIONAL STUDY
}

\author{
$\underline{\text { Paul Noone }}^{1}$, Guido Pagnacco ${ }^{2,3}$, and Elena Oggero ${ }^{2,3}$ \\ ${ }^{1}$ Small Street Clinic, Hampton, Melbourne, Australia \\ ${ }^{2}$ Electrical and Computer Engineering Department, University of Wyoming, Laramie, WY, USA \\ ${ }^{3}$ Vestibular Technologies, LLC, Cheyenne, WY, USA
}

Corresponding Author: Paul Noone

Small Street Clinic1a/10 Small St, Hampton, 3188, Melbourne, Australia; www.smallstreetclinic.com

Tel: +61421056110

Email: plnoone1@gmail.com

https://doi.org/10.34107/BiomedSciInstrum.57.0145

\begin{abstract}
Central Sensitization (CS) is postulated as a central explanation of chronic pain. Clinical researchers recommend that therapists screen for CS to avoid diagnostic confusion and improve the allocation of appropriate clinical resources in primary care settings when managing chronic pain patients. However, the percentage of patients presenting with CS to a chiropractic practice has not been found in the literature. This study had two objectives: to use the Central Sensitization Inventory (CSI) to screen for and identify the proportion and characteristics of consecutive new patients with chronic pain conditions and medically unexplained symptoms who are experiencing CS; and to determine if there were significant clinical relationships between patient characteristics (age, sex, BMI, complaint type and duration, balance issues and presence of comorbid overlapping CS syndromes) and CSI scores. Results indicated that 1 in 5 adult new patients may have experienced CS. ANOVA analysis revealed significant difference between fibromyalgia and chronic spinal pain patients; significant difference due to subjective dizziness; significant difference between the number of positive answers in the CSI-Part B for the CSI $>40$ subjects and subjects with 2 or more positive answers on CSI Part B compared to those with none/one positive answer. Chiropractors should consider using a validated CS screening tool, such as the CSI, for all new patients, and implementing adjunctive, evidence-based CS clinical management strategies.
\end{abstract}

Keywords: Practice based clinical research, Chiropractic, Chronic pain, Central sensitization, Central sensitization inventory

\section{INTRODUCTION}

Chiropractors treat patients who have overlapping chronic pain conditions (OCPC) and medically unexplained symptoms (MUS) [1-4]. OCPC and MUS conditions reported in the scientific literature include: fibromyalgia (FM), chronic non-specific low back pain (CNSLBP), chronic non-specific neck pain (CNSNP), chronic tension-type headache (CTTH), unexplained dizziness (UD) and irritable bowel syndrome (IBS) amongst others [5-8]. Comorbid symptoms such as anxiety and depression, subjective dizziness/postural instability and autonomic dysfunction often accompany these conditions [9-12].

Central Sensitization (CS) is postulated as a central explanation of chronic pain [13]. CS is defined as "...increased responsiveness of nociceptive neurons in the central nervous system to their normal or sub-threshold afferent input" [14]. Numerous authors have proposed neurobiological explanations underpinning CS [15-16]. Centrally sensitized chronic pain is identified as a significant burden on global economic, social and clinical resources [17]. Clinical researchers suggest that MUS have CS as an underlying aetiology [6]. Some authors propose that subjective dizziness complaints such as Persistent 